\title{
Les polices nationales et l'unification européenne, enjeux et interactions. Remarques introductives
}

René Lévy et Dominique Monjardet

\section{(2) OpenEdition \\ 12 Journals}

Édition électronique

URL : http://journals.openedition.org/conflits/896

DOI : $10.4000 /$ conflits.896

ISSN : $1777-5345$

Éditeur :

CCLS - Centre d'études sur les conflits lilberté et sécurité, L'Harmattan

Édition imprimée

Date de publication : 1 décembre 2002

ISBN : 2-7475-4291-2

ISSN : 1157-996X

\section{Référence électronique}

René Lévy et Dominique Monjardet, « Les polices nationales et l'unification européenne, enjeux et interactions. Remarques introductives », Cultures \& Conflits [En ligne], 48 I hiver 2002, mis en ligne le 05 mai 2003, consulté le 30 mars 2021. URL : http://journals.openedition.org/conflits/896 ; DOI : https:// doi.org/10.4000/conflits.896

Ce document a été généré automatiquement le 30 mars 2021.

Creative Commons License 


\title{
Les polices nationales et l'unification européenne, enjeux et interactions. Remarques introductives
}

\author{
René Lévy et Dominique Monjardet
}

1 L'intérêt suscité par le développement de multiples formes de coopération interétatiques dans le domaine policier donne naissance principalement à deux orientations de recherche qui ne saturent pas le champ. D'une part est développée l'analyse comparée des différents systèmes de police. D'autre part les dispositifs actuels de coopération internationale, tout particulièrement européens (Trévi, Schengen, Europol, etc.), font l'objet d'études nombreuses ${ }^{1}$. Par contre l'analyse de l'interaction entre les logiques internes de fonctionnement des systèmes policiers nationaux et ces dispositifs de coopération internationale est encore en friche et c'est à cette question que les textes réunis ci-après s'efforcent d'apporter quelques éléments de réponse ${ }^{2}$.

Dans quelle mesure des spécificités nationales marquées facilitent ou handicapent-elles les coopérations décidées au niveau politique multinational, sous quelles formes à l'inverse les dispositifs pratiques de coopération influent-ils sur le fonctionnement propre des appareils policiers locaux? La question n'est pas entièrement nouvelle : de tout temps les polices d'Etat et notamment leurs branches politiques ont coopéré dans le renseignement et le contrôle de groupes identifiés comme subversifs ou terroristes. Depuis 1923, Interpol concrétise un souci de coopération dans la lutte contre la grande criminalité, depuis deux décennies la lutte contre le trafic des stupéfiants a pris une dimension internationale marquée. Dans tous ces cas cependant l'objet était précisément cerné et les outils de la coopération limités à des segments bien identifiés des appareils policiers, peu susceptibles donc d'affecter en profondeur les caractéristiques significatives de chacun des systèmes policiers concernés. Il en va autrement lorsque tant sous l'effet de la transnationalisation croissante des flux (de personnes et de biens) que par la volonté politique de créer un espace européen, les 
objets et les outils de la coopération en matière de police et de sécurité se généralisent. Sont alors concernés non plus seulement des cellules policières spécialisées (Offices, bureaux ou correspondants ad hocle plus souvent directement rattachés au centre), mais des branches entières de l'appareil policier ou des modes de fonctionnement, procédures, techniques, répandus dans l'ensemble de chaque système. Illustrent ces nouveaux mécanismes aussi bien la question des migrations transfrontalières, qui posent aujourd'hui avec acuité le problème de l'harmonisation des techniques de contrôle, que celui du maintien de l'ordre qui, moins explicitement mais tout aussi sûrement, conduit - à l'occasion, par exemple, des grandes manifestations sportives - à une diffusion de techniques spécialisées et à un échange massif d'informations. En sens inverse, la disparité des législations nationales à l'égard des consommateurs de différentes catégories de stupéfiants a des effets directs sur l'action policière dans chacun des pays entre lesquels ces consommateurs circulent librement.

3 L'ensemble de textes présentés ci-après a donc pour objet non pas la coopération internationale en tant que telle, mais en tant qu'elle produit un espace de rencontre, d'influence, de confrontation, voire de concurrence entre des systèmes de police différents, caractérisés chacun par ses enjeux propres, son organisation, ses principes juridiques. Instruments de souveraineté interne par excellence, ces systèmes ont été développés historiquement de façon jalousement autonome, et dans une grande distance, si ce n'est une grande méfiance à l'égard de toute expérience étrangère, considérée comme non pertinente par définition. Il s'agit ici d'examiner dans quelle mesure la coopération internationale les met en mouvement et avec quels effets.

4 Les articles de Hartmut Aden, Azilis Maguer, Paul Swallow et James Sheptycki sont de portée différente: deux d'entre eux - ceux de Aden et de Shepticky - embrassent l'ensemble du champ de la sécurité, mais en développant des points de vue assez distincts; le premier s'attache à un examen systématique des effets internes de la coopération européenne tandis que second s'interroge essentiellement sur les difficultés nouvelles que la configuration contemporaine des systèmes policiers oppose à l'impératif démocratique du contrôle des polices. De leur côté, les articles de Maguer et Swallow ont un caractère plus monographique : le premier procède à une analyse serrée des répercussions de la coopération entre services français et allemands à la frontière entre les deux pays, sous les angles de l'institution, de la profession et de l'organisation; le second propose le point de vue d'un cadre de la police britannique engagé dans la coopération policière.

5 Pour évaluer les effets des dispositifs internationaux, et plus spécialement européens, sur les organisations policières internationales, un certain nombre de distinction s'imposent. Comme le rappelle Hartmut Aden, qui voit dans le système policier européen un système émergent de multi-level governance( ce qu'on pourrait traduire par "gouvernement à niveaux multiples »), tous les services de police et tous les policiers ne participent pas au même titre à la coopération : que l'on songe, par exemple, à la distance qui existe entre le gardien de la paix d'une ville moyenne (non-frontalière) et l'officier de liaison européen ou le fonctionnaire affecté à un commissariat conjoint ; leur seul point commun, c'est vraisemblablement l'utilisation d'une base de données qui intègre de manière croissante la dimension européenne.

6 A cet égard, deux dimensions s'imposent à la réflexion. La première renvoie à la distinction des "métiers" de la sécurité, tels qu'ils se présentent aujourd'hui à un observateur qui ne s'arrête pas à une définition juridico-administrative de la police. On 
y trouve, naturellement, les trois grands «métiers policiers » : police d'ordre, police criminelle et police urbaine ${ }^{3}$, distinction qui s'applique mutatis mutandis à la police nationale comme à la gendarmerie nationale, dans le cas français, mais est également repérable dans tous les dispositifs policiers étrangers. Cependant, au plan européen - et aussi, du reste et de façon croissante, au plan national - il faut prendre en considération l'administration douanière, dont les pouvoirs et les pratiques tendent à se rapprocher de celles des polices « ordinaires ». Il faut également prendre en considération, comme le font clairement Aden et Sheptycki ci-après, le secteur de la sécurité privée, dont la croissance se caractérise par deux tendances lourdes, une interpénétration avec le secteur public (comme force de substitution ou de complément) et une internationalisation croissante.

7 La seconde dimension renvoie aux différents aspects de l'institution policière (au sens large indiqué ci-dessus) susceptibles d'être affectés par l'européanisation: l'organisation, les personnels, les pratiques. C'est à l'intersection de ces deux axes qu'il faut rechercher les effets de la coopération européenne.

Les effets sur l'organisation des polices

8 Les effets de la coopération européenne sur l'organisation policière interne peuvent être analysés à deux niveaux : globalement, au niveau de l'organisation d'ensemble du dispositif policier, et agence par agence.

9 Selon Aden ${ }^{4}$, la coopération policière exerce un effet de centralisation sur les systèmes policiers, dans la mesure où elle impose à chaque pays de créer des organes spécifiques de liaison avec leurs homologues. C'était déjà le cas lorsque le principal instrument de coopération était Interpol, avec la création d'un bureau de liaison spécifique dans chacun des pays participants. Schengen n'a fait qu'accentuer cette tendance, chaque pays étant amené à désigner une institution servant d'interface entre les services de police de base et le réseau de coopération et d'information européen. Il en a fréquemment résulté des tensions internes lorsque plusieurs structures de police pouvaient prétendre jouer ce rôle et ceci, indépendamment du caractère plus ou moins centralisé des institutions policières. En effet, ces tensions sont observables aussi bien dans des pays où elles sont relativement décentralisées (Allemagne, Pays-Bas, Royaume-Uni) que dans des pays relativement centralisés (France). Dans le premier cas, cependant, ces tensions ne sont pas toujours de même nature : comme l'explique Aden, en Allemagne et au Pays-Bas, ce sont les autorités régionales, accoutumées à une large autonomie, qui renâclent à la désignation d'un chef de file national ; inversement, selon Swallow, l'établissement du NCIS britannique a été perçu par les polices régionales comme un moyen de limiter la prééminence traditionnelle de Scotland Yard, c'est-àdire de la police londonienne.

10 En France, pays généralement perçu comme le parangon de la centralisation policière, mais qui dispose en réalité de plusieurs agences centralisées plus ou moins rivales ${ }^{5}$, la désignation d'un chef de file dans le domaine de la coopération internationale a donné lieu à des luttes acharnées, qui n'ont pas toujours tourné à l'avantage des mêmes : dans le cas du système Schengen, la police nationale a été désignée comme chef de file, au détriment de la gendarmerie nationale; par contre, en ce qui concerne la lutte contre le blanchiment, elle a été supplantée par les services du ministère des Finances, tutelle traditionnelle du système bancaire. Toutefois la prédominance de telle agence au niveau supérieur ne garantit pas sa plus grande efficacité sur le terrain. Maguer montre clairement, par exemple, que c'est au niveau local que se prennent les initiatives en 
matière de coopération, que l'aptitude des instances centrales de chaque organisation à les relayer et à les défendre auprès de l'autorité politique est variable et qu'en la matière, la gendarmerie est plus efficace que la police nationale. En ce sens, la thèse centralisatrice d'Aden doit être soigneusement nuancée. Où il apparait à nouveau que la plasticité, ou capacité d'adaptation des structures policières, est infiniment plus grande quand le moteur est l'intérêt professionnel policier que lorsqu'il s'agit de mettre en œuvre une réforme imposée par le politique.

11 Par ailleurs, même si certains dispositifs de coopération poussent à la centralisation, ils ne supplantent pas les dispositifs pré-existants, dont la multiplicité permet à chaque institution particulière de préserver sa marge de manœuvre en nouant des relations privilégiées avec les institutions homologues des autres pays. C'est peut-être même davantage le cas, comme le montre très clairement Maguer, lorsque les différentes composantes de plusieurs systèmes policiers nationaux sont réunies dans un organe de coopération commun, comme le Centre franco-allemand de coopération policière et douanière $(\mathrm{CPD})$ localisé en Allemagne, près de la frontière avec la France, et où chacun peut faire jouer à son profit la concurrence entre ses partenaires étrangers. De même, comme le signale Swallow, Schengen n'a pas fait disparaître NEBEDEAG-Pol à la frontière germano-belgo-néerlandaise, ni l'unité de liaison de la police du Kent et ses ramifications internationales liées au tunnel sous la Manche, et il ne s'agit là que de deux structures de coopération parmi une vingtaine d'autres d'importance variable, dont certaines sont peu visibles - qui connait, par exemple, la European Association of Airport and Seaport Police (EAASP) ou le Colloque des polices ferroviaires (COLPOFER) ? - et qui composent les "archipels policiers ${ }^{6}$. Swallow, dont le point de vue est celui d'un praticien engagé dans la coopération policière, fait cependant preuve d'un certain optimiste, lorsqu'il estime que des organes de coordination tels que le NCIS britannique, embrassant, outre les polices régionales, les douanes et les services de l'immigration, permettent de briser certaines barrières corporatives et d'habituer les fonctionnaires des différentes agences à travailler de concert.

12 La multiplicité même des dispositifs de coopération ou d'échange complique singulièrement la mesure de leurs effets sur les différentes agences policières prises isolément. Il est clair cependant que l'on a assisté à des réorientations massives de certaines agences, en particulier celles dont l'activité était centrée sur les frontières, sauf peut-être au Royaume-Uni pour des raisons qui tiennent autant à la géographie qu'à la méfiance traditionnelle envers le Continent, comme le montre Swallow. Dans le cas français, on sait que la police aux frontières et la douane ont été redéployées vers les « frontières externes » de l'Union, c'est-à-dire les aéroports et les régions côtières et ont vu leurs zones d'intervention étendues à l'ensemble du territoire. De manière plus significative, la douane est devenue un acteur pleinement reconnu de la sécurité intérieure et européenne ${ }^{7}$. Inversement, l'organisation de la police urbaine est largement préservée des effets de l'européanisation policière, même dans les secteurs frontaliers. Il en va de même de l'autre branche de la police en uniforme que constituent les forces spécialisées dans le maintien de l'ordre, ce qui ne veut pas dire que leurs techniques n'en sont pas affectées.

Les effets sur les techniques et pratiques policières

13 Les effets de la coopération européenne sur les techniques et les pratiques policières sont très variables. De ce point de vue on pourrait schématiquement établir une échelle 
dont le barreau inférieur serait la police urbaine et le niveau supérieur les services à vocation frontalière.

Dans la plupart des cas, comme l'indique Aden, le seul point de rencontre entre le policier en tenue et les dispositifs européens, c'est la consultation d'un fichier qui se trouve désormais être alimenté par des données d'autres pays ou interconnecté au Système Informatique Schengen. Ce changement n'est pas négligeable, mais il ne modifie pas réellement le travail de l'intéressé, qui du reste ne le perçoit pas nécessairement. La situation est différente en zone frontalière, comme le montre Maguer, puisque les policiers peuvent être amenés à exercer, dans certaines conditions, un droit de suite sur le territoire d'un État voisin.

En matière de maintien de l'ordre, on peut également déceler des changements liés à des transferts de savoir-faire entre polices plus ou moins habituées à affronter des risques spécifiques. C'est notamment le cas en matière de gestion des grands évènements sportifs où la concertation et l'échange de renseignements entre polices $\mathrm{du}$ lieu de l'évènement et polices des lieux de provenance des supporters est devenue courante ${ }^{8}$.

16 C'est sans doute dans le domaine de la police criminelle que les changements induits par la coopération policière sont le plus nets, sans pour autant s'étendre à l'ensemble des services. L'évolution est particulièrement sensible dans le domaine de l'information (avec l'accès aux fichiers européens, ou l'accès indirect à des fichiers étrangers, au sein des commissariats conjoints), de l'uniformisation de certaines techniques d'identification et de la généralisation des techniques dites "proactives", notamment clandestines (undercover). Comme le souligne Aden, la coordination de telles opérations est l'une des fonctions privilégiées d'Europol. Dans ce domaine, le fait le plus notable est toutefois le brouillage des distinctions traditionnelles entre la police criminelle au sens classique du terme et les autres agences, comme les douanes, voire les services de renseignement, comme le signale Swallow à propos du Royaume-Uni et comme on peut l'observer avec la relative réorientation des missions assignées aux Renseignements généraux français vers la criminalité organisée (réorientation vraisemblablement remise en cause par les attentats du 11 septembre 2000).

17 En ce qui concerne la douane, il est patent, dans le cas de la France, que la disparition des frontières intérieures à l'UE - accentuant le « découplage entre frontière et lieu du contrôle $»^{9}$ - a amené cette institution à repenser ses méthodes, à développer sa coopération avec ses homologues étrangers et à rechercher une assimilation de ses pouvoirs à ceux des services de la police judiciaire, au nom de l'uniformisation européenne des compétences douanières.

18 Il resterait enfin à développer la recherche dans un champ insuffisamment pris en compte, mais dont ici encore les indications d'Aden montrent tout l'intérêt : les effets croisés de l'action policière et de l'harmonisation judiciaire. Deux dimensions distinctes peuvent en être signalées. D'une part le développement progressif d'un code de procédure pénale européen, sous le double effet formel de la jurisprudence de la Cour européenne des droits de l'homme, et informel de la coopération quotidienne dans les zones frontalières. D'autre part, l'asymétrie entre les coordinations policières et judiciaires: Maguer montre bien que lorsque les relations entre services de police anticipent très fortement sur celles entre les services judiciaires correspondants (parquets et juges d'instruction), ces derniers sont de plus en plus mal armés pour encadrer l'action policière. C'est bien plutôt celle-ci, mieux outillée techniquement, qui 
va «conseiller et coordonner " les relations transfrontalières des services judiciaires. L'avenir dira si la mise en place d'Eurojust est susceptible d'équilibrer ces rapports.

Les effets sur les personnels

Dans ce domaine, l'effet le plus visible de l'européanisation est l'émergence d'une strate d'agents fortement impliqués dans les processus de coopération et qui présentent des caractéristiques bien particulières, qui ont été étudiés notamment par Didier Bigo ${ }^{10}$ : études supérieures impliquant des séjours à l'étranger, maitrise de plusieurs langues, carrières atypiques... Il est trop tôt, par ailleurs, pour évaluer la portée du projet de Collège européen de police destiné à fournir un ensemble de références communes aux cadres supérieurs des polices européennes.

Le séminaire dont sont issus ces textes s'est tenu en mars 2001, et il est encore trop tôt pour que des recherches sérieuses nous informent sur les conséquences, pour la coopération policière internationale, des événements du 11 septembre. Deux traits sont cependant notables ${ }^{11}$. En premier lieu, l'événement a donné naissance, dans pratiquement tous les pays occidentaux, à une abondante production législative destinée à renforcer à la fois les dispositifs de protection nationaux (dans les domaines du transport aérien et du contrôle des étrangers, notamment), et les moyens de lutte contre le terrorisme. Dans ce dernier champ des mesures de coopération internationale spécifiques ont été amplement développées. On peut donc en attendre un renforcement des formes traditionnelles de coopération: partage des fichiers, renforcement des dispositifs d'écoute et interception des communications, échanges d'information, multiplication des officiers de liaison, développement et simplification des mécanismes d'entraide judiciaire, etc.

21 Mais les mesures très techniques prises dans ces différents domaines ne suffisaient sans doute pas à témoigner devant les opinions publiques de la vigilance et de la mobilisation des pouvoirs, les lois alors votées ont été ainsi complétées par des dispositions de tous ordres n'ayant le plus souvent qu'un rapport très lointain avec le terrorisme international. Le cas de la loi Vaillant en France est ici particulièrement illustratif : le texte initial déposé en mars 2001 est "relatif à la sécurité quotidienne ", et entend répondre à la montée de la délinquance enregistrée par la statistique nationale en 2000. A la suite du 11 septembre, le gouvernement y adjoint, par amendements, treize dispositions nouvelles visant à « mieux lutter contre le terrorisme ». En ajoutant que la lutte contre le terrorisme implique de s'attaquer « au financement des trafics qui l'alimentent - trafics d'armes et trafic de stupéfiants ${ }^{12}$, on en étend le champ à l'infini. C'est ainsi que vont être satisfaites de très anciennes revendications policières, constamment rejetées jusqu'alors, telles la possibilité de fouiller les coffres des automobiles, ou d'opérer des perquisitions de nuit ${ }^{13}$.

22 Une étude spécifique serait nécessaire pour vérifier une hypothèse adjacente : dans ces conjonctures où les polices de chaque pays s'empressent de faire avancer auprès de leurs pouvoirs respectifs leurs revendications traditionnelles, la comparaison internationale joue un rôle croissant. Pourquoi refuser aux policiers français une prérogative dont jouissent déjà - et avec bonheur, nécessairement - les policiers anglais (ou belges, ou danois), et réciproquement? Se développerait alors, sous l'influence des diverses organisations internationales de policiers, ce même mécanisme d'effet de cascade qui généralise à tous l'avancée locale de chacun. 


\section{NOTES}

1. . Par exemple : Anderson M., den Boer M. (Eds.), Policing across national boundaries, London-New York, Pinter Publishers. 1994 ; Bigo D., Polices en réseaux, l'expérience européenne, Paris, Presses de Sciences Po, 1996 ; Sabatier M., La coopération policière européenne, Paris, L'Harmattan, 2001.

2. . Les quatre articles de ce numéro sont issus de communications présentées au séminaire du Groupe Européen de Recherche sur les Normativités (GERN-CNRS) intitulé Questions de polices/Policing Matters, le 23 mars 2001 (MSH, Paris). Ce séminaire est animé par Jean-Marc Berlière, Clive Emsley, René Lévy et Dominique Monjardet. 3. . Monjardet D., Ce que fait la police. Sociologie de la force publique, Paris, La découverte, 1996.

4. . La simple mention du nom d'un auteur renvoie à l'article correspondant publié ciaprès.

5. . Dans la typologie des polices élaborée par Bailey, il s'agit d'une «structure centralisée à polices multiples coordonnées » (Bayley D.H., Patterns of Policing. A Comparative International Analysis, New Brunswick, N.J., Rutgers U.P., 1985, p. 59). 6. . Selon l'heureuse formule de Didier Bigo.

7. . Rôle avalisé par la loi d'orientation et de programmation relative à la sécurité du 21 janvier 1995.

8. Voir le numéro spécial Football, ombres au spectacle des Cahiers de la sécurité intérieure, 1996, 26.

9. . Selon la formule de C. Paulard-Lanapats, (Les douaniers français, la frontière et la construction européenne, Paris, IHESI, 1999, p.25) qui insiste également sur le rôle de l'Organisation Mondiale des Douanes dans le transfert des informations et des techniques (p. 28 s.). Voir aussi Lévy R. «Les livraisons surveillées de stupéfiants : légaliser pour mieux contrôler? » Questions pénales, 2002, 15, 5 ; et Lévy R., Légaliser pour mieux contrôler? Le cas des livraisons surveillées de stupéfiants et la loi française du 19 décembre 1991, Guyancourt, CESDIP, 2003 (à paraître); Kletzlen A., La place de l'administration des douanes dans la régulation des DEFT, Guyancourt, CESDIP, 2002. Voir aussi le prochain numéro de Cultures \& Conflits sur le visa Schengen sous la responsabilité de Didier Bigo et Elspeth Guild.

10. . Bigo D., "Liaison officers in Europe : new officers in the European security field ", in Shepticky J. W. E., (Ed.), Issues in transnational policing, Londres, New York, Routledge, 2000.

11. . En attendant des études plus systématiques, on pourra se référer au panorama dressé par Courrier International, $n^{\circ} 583$ du 3 au 9 janvier 2002, au rapport 2002 d'Amnesty International, aux rapports et à l'article de Mary Robinson, hautcommissaire des Nations Unies aux droits de l'homme (Le Monde 15/06/02). 12. . Vaillant D., ministre de l'Intérieur, Sénat, séance du 16 octobre 2001. 13. . Monjardet D., Le terrorisme international et la cage d'escalier. La sécurité publique dans le débat politique en France, 2000-2003, Communication au colloque En quête de sécurité de la Commission du droit du Canada (Montréal, 19-22 février 2003). 
INDEX

Index géographique : Europe

Mots-clés : construction européenne, police, coopération internationale

Thèmes : EUROPOL 